\title{
Transmission-Blocking Vaccines: Focus on Anti-Vector Vaccines against Tick-Borne Diseases
}

\author{
Girish Neelakanta $\cdot$ Hameeda Sultana
}

Received: 26 August 2014 / Accepted: 15 October 2014/Published online: 12 December 2014

(C) The Author(s) 2014. This article is published with open access at Springerlink.com

\begin{abstract}
Tick-borne diseases are a potential threat that account for significant morbidity and mortality in human population worldwide. Vaccines are not available to treat several of the tick-borne diseases. With the emergence and resurgence of several tick-borne diseases, emphasis on the development of transmission-blocking vaccines remains increasing. In this review, we provide a snap shot on some of the potential candidates for the development of antivector vaccines (a form of transmission-blocking vaccines) against wide range of hard and soft ticks that include Ixodes, Haemaphysalis, Dermacentor, Amblyomma, Rhipicephalus and Ornithodoros species.
\end{abstract}

Keywords Transmission-blocking vaccine ·

Anti-vector vaccine $\cdot$ Ixodes - Dermacentor $\cdot$ Amblyomma . Haemaphysalis $\cdot$ Rhipicephalus $\cdot$ Ornithodoros species

\section{Introduction}

Arthropod-borne diseases account for severe morbidity and mortality in worldwide human population (Githeko et al. 2000; Hill et al. 2005). Based on the disability-adjusted life year (DALY) estimates, infections associated with onesixth of the human population are due to arthropod-borne diseases (Hill et al. 2005; WHO 2004). World Health Organization (WHO) reports malaria to be the leading vector-borne disease in the world followed by

G. Neelakanta $(\bowtie) \cdot$ H. Sultana $(\bowtie)$

Center for Molecular Medicine, Department of Biological

Sciences, Old Dominion University, Norfolk, VA 23529, USA

e-mail: gneelaka@odu.edu

H. Sultana

e-mail: hsultana@odu.edu
Leishmaniasis, Trypanosomiasis, Yellow fever, Dengue, Chagas disease and Japanese Encephalitis (Hill et al. 2005; WHO 2004, 2013, 2014). The recent epidemics of historically recognized diseases such as Tick-borne encephalitis, Kyasanur forest disease, Crimean-Congo hemorrhagic fever and Rocky mountain spotted fever suggests increase in the scope and magnitude of tick-borne diseases world wide (Demma et al. 2006; Maltezou et al. 2010; Pattnaik 2006; Randolph 2008). Even though tick-borne diseases are considered pale in comparison to the other arthropodborne diseases, the steady increase in the annual incidence of some of the tick-borne diseases such as Lyme disease, human anaplasmosis and human monocytic ehrlichiosis implies a potential threat to human health (CDC 2014). Some of the important tick-borne diseases that occur worldwide are listed in Table 1 . No vaccines or effective therapies are available to treat several of these important vector-borne diseases.

There are about 5-19 million species of arthropods existing in the world of which some serve as vectors for various pathogens that cause diseases in humans (Ødegaard 2000). Arthropods have become most successful to serve as competent vectors for disease transmission due to their capacity of biting host, ingesting blood meal from hosts and permitting pathogen survival in them for a longer period of time (Desenclos 2011; Goddard 2008). Several studies have reported use of live-attenuated parasite vaccine candidates to control arthropod-borne disease pathogenesis in various animal models (Barry et al. 2009; Callow 1978; Conlan 2011; Gardner and Ryman 2010; Heinz and Stiasny 2012; Orlinger et al. 2011; Reed et al. 2014; Sultana et al. 2009; Wang et al. 2014a; Yun and Lee 2014). However, limited of them are successful and approved for human use (Conlan 2011; Gardner and Ryman 2010; Orlinger et al. 2011; Yun and Lee 2014). 
Table 1 Worldwide tick-borne diseases

\begin{tabular}{|c|c|c|c|}
\hline Tick-borne diseases & Agent & Tick species & Tick family \\
\hline \multirow[t]{12}{*}{ Lyme Borreliosis } & \multirow[t]{3}{*}{ Borrelia burgdorferi } & Ixodes scapularis & Ixodidae \\
\hline & & I. dentatus & Ixodidae \\
\hline & & I. pacificus & Ixodidae \\
\hline & \multirow[t]{3}{*}{ B. afzelii } & I. ricinus & Ixodidae \\
\hline & & I. persulcatus & Ixodidae \\
\hline & & I. nipponensis & Ixodidae \\
\hline & \multirow[t]{6}{*}{ B. garinii } & I. ricinus & Ixodidae \\
\hline & & I. uriae & Ixodidae \\
\hline & & I. hexagonus & Ixodidae \\
\hline & & I. trianguliceps & Ixodidae \\
\hline & & I. persulcatus & Ixodidae \\
\hline & & Haemaphysalis longicornis & Ixodidae \\
\hline \multirow[t]{2}{*}{ Human Anaplasmosis } & \multirow{2}{*}{ Anaplasma phagocytophilum } & I. scapularis & Ixodidae \\
\hline & & I. pacificus & Ixodidae \\
\hline Human Babesiosis & Babesia microti & I. scapularis & Ixodidae \\
\hline \multirow[t]{5}{*}{ Tularemia } & \multirow[t]{5}{*}{ Francisella tularensis } & I. dentatus & Ixodidae \\
\hline & & I. ricinus & Ixodidae \\
\hline & & Dermacentor variabilis & Ixodidae \\
\hline & & D. andersoni & Ixodidae \\
\hline & & Amblyomma americanum & Ixodidae \\
\hline \multirow[t]{4}{*}{ Q-fever } & \multirow[t]{4}{*}{ Coxiella burnetii } & I. dentatus & Ixodidae \\
\hline & & I. trianguliceps & Ixodidae \\
\hline & & Rhipicephalus sanguineus & Ixodidae \\
\hline & & A. americanum & Ixodidae \\
\hline \multirow[t]{3}{*}{ Japanese spotted fever } & \multirow[t]{3}{*}{ Rickettsia japonica } & I. ovatus & Ixodidae \\
\hline & & D. taiwanensis & Ixodidae \\
\hline & & H. flava & Ixodidae \\
\hline Queensland tick typhus & R. australis & I. holocyclus & Ixodidae \\
\hline \multirow[t]{2}{*}{ Tick-borne encephalitis } & \multirow[t]{2}{*}{ Tick borne encephalitis virus } & I. ricinus & Ixodidae \\
\hline & & I. persulcatus & Ixodidae \\
\hline Boutonneuse fever & R. conorii & $R$. sanguineus & Ixodidae \\
\hline \multirow[t]{2}{*}{ Ehrlichiosis } & Ehrlichia chaffeensis & A. americanum & Ixodidae \\
\hline & E. ewingii & A. americanum & Ixodidae \\
\hline \multirow[t]{4}{*}{ Rocky mountain spotted fever } & \multirow[t]{4}{*}{ R. rickettsii } & A. canjennense & Ixodidae \\
\hline & & D. variabilis & Ixodidae \\
\hline & & D. andersoni & Ixodidae \\
\hline & & $R$. sanguineus & Ixodidae \\
\hline African tick-bite fever & R. africae & A. hebraeum & Ixodidae \\
\hline Siberian tick typhus & R. sibirica & D. nuttalli & Ixodidae \\
\hline \multirow[t]{3}{*}{ Relapsing fever } & B. duttoni & Ornithodoros moubata & Argasidae \\
\hline & B. hermsii & O. hermsi & Argasidae \\
\hline & B. turicatae & O. turicata & Argasidae \\
\hline African relapsing fever & B. crocidurae & O. erraticus sonrai & Argasidae \\
\hline
\end{tabular}

Therefore, effective strategies need to be developed in order to combat both arthropods and pathogens. Of many strategies (Coutinho-Abreu et al. 2009; Oliveira et al. 2009; Thomas and Read 2007; Valenzuela 2004a, b), development of transmission-blocking vaccines has provided a significant leap that has moved research in this field forward for clinical trials (Malkin et al. 2005; Saul et al. 2007; Wu et al. 2008).

Anti-vector vaccines are a type of transmission-blocking vaccines aimed to target vector molecules to block pathogen 
transmission from arthropods to mammalian hosts (Billingsley et al. 2008; Coutinho-Abreu and Ramalho-Ortigao 2010; Coutinho-Abreu et al. 2009; Oliveira et al. 2009; Valenzuela 2004a). Several features are important to be considered for the development of anti-vector vaccines for humans (de la Fuente and Merino 2013; Merino et al. 2013). First, candidate molecule should be critical for vectorpathogen interaction. Disruption of the candidate molecule by gene knock-out/down, RNA interference or antibody blocking should affect acquisition or transmission or replication of pathogen inside vector. Second, the primary amino acid sequence of the candidate molecule should be highly conserved among different isolates of that species to facilitate development of unique antigen for vaccine design. Third, candidate molecule should provide high antibody titer upon injection into humans to block pathogen transmission from vectors. Fourth, candidate molecule should be compatible with different adjuvants to effectively induce immune responses in humans. Lastly, candidate molecule should not result in exaggerated immune responses leading to immune-related disorders in humans.

The basic proposed strategy on the effect of anti-vector vaccination in humans is illustrated in Fig. 1. For instance, in the Northeastern part of the United States, Ixodes scapularis ticks transmit Borrelia burgdorferi the causative agent of Lyme disease, Anaplasma phagocytophilum the agent of human anaplasmosis and Babesia microti the agent of human Babesiosis (Table 1) (Anderson and Magnarelli 2008). In nature, I. scapularis larval ticks get infected with these pathogens upon feeding on infected vertebrate hosts (Sonenshine and Roe 2014). Infected larval ticks molt into nymphs. Humans accidently become infected upon bite by an infected nymph carrying these pathogens (Fig. 1). Three types of anti-vector vaccine development strategies can be proposed to protect humans from being infected. First, anti-vector vaccine can be designed to degrade pathogens inside ticks that subsequently make ticks free of pathogens (Fig. 1). Second, antivector vaccine can be developed against one of the vector molecule to prevent transmission of pathogens to humans (Fig. 1). Third, anti-vector vaccines can be designed to degrade or affect physiology of ticks upon ingestion of a blood meal. Collectively, these proposed strategies would significantly improve development of potential therapeutic targets to treat and/or control several of the human vectorborne diseases.

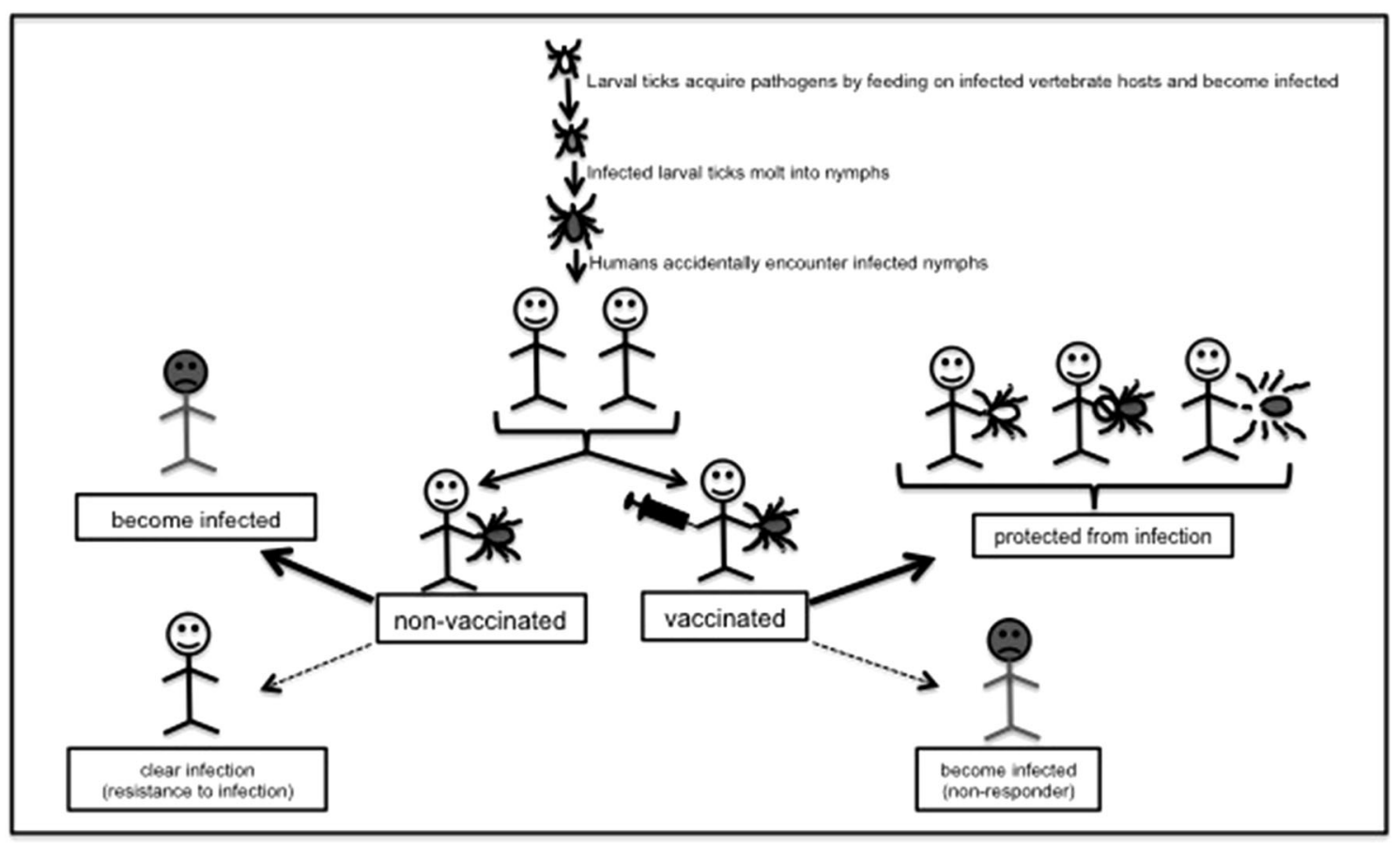

Fig. 1 Anti-vector vaccines. Schematic representation of the proposed strategy on the effect of anti-tick vaccination in humans is shown. Larval ticks become infected by feeding on infected vertebrate hosts and molt into nymphs. Nymphs transmit pathogens to humans. Several scenarios might be envisioned with regard to the effect of antitick vaccine administration in natural human population. Nonvaccinated humans bitten by infected ticks may become infected and develop disease symptoms or remain uninfected by natural resistance.
On the other hand, vaccinated individuals might be protected from infection or may still become infected due to poor responsiveness to the vaccine. As shown in the illustration, three types of vaccine strategies can be considered. First, anti-vector vaccine can be designed to degrade pathogens inside ticks. Second, anti-vector vaccine can be designed to target vector molecules to prevent pathogen transmission. Third, anti-vector vaccine can be designed to target vector molecules to degrade or affect physiology and survival of ticks 
In this review, we focus on some of the important findings in the field of anti-vector vaccines against wide range of hard and soft ticks that include Ixodes, Haemaphysalis, Dermacentor, Amblyomma, Rhipicephalus and Ornithodoros species. Due to our focus on providing a "snap shot" with certain level of subjectivity of including studies from both hard and soft ticks, several of the other important findings in this field are not included.

\section{Anti-Ixodes Vaccine Candidates}

Several of the studies have reported immunization of animals with tick antigens to study the effect of vaccination on pathogen transmission and acquisition. Active immunization of mice with Salp15, a $15-\mathrm{kDa}$ secreted salivary gland protein from $I$. scapularis showed substantial protection (60\%) from tick-borne Borrelia (Dai et al. 2009). Salp15 protein has several immunosuppressive properties (Juncadella and Anguita 2009). It inhibits $\mathrm{CD}^{+} \mathrm{T}$ cell activation, complement activity and dendritic cell activation (Juncadella and Anguita 2009). B. burgdorferi coats itself with Salp15 protein during exit from ticks (Ramamoorthi et al. 2005). This coating prevents evasion of immune responses against spirochetes upon entry into the mammalian host (Ramamoorthi et al. 2005). Interaction of Salp15 with B. burgdorferi OspC is critical during this process (Ramamoorthi et al. 2005). Immunization of mice with Salp25D, a 25-kDa salivary gland protein from $I$. scapularis reduced spirochete acquisition by ticks to threefold in comparison to the nonimmunized controls (Narasimhan et al. 2007). Salp25D has significant homology to peroxiredoxins antioxidants that has been shown to play significant role in protecting $B$. burgdorferi from reactive oxygen produced by neutrophils (Narasimhan et al. 2007). Blockade of gutspecific protein TROSPA (tick receptor for outer surface protein A) by TROSPA-antisera showed significant reduction (75\%) of $B$. burgdorferi adherence to tick gut in vivo that subsequently prevented colonization and transmission of spirochete from ticks to mice (Pal et al. 2004). Interaction of TROSPA with B. burgdorferi Outer surface protein A (OspA) is critical for colonization of spirochetes in the gut (Pal et al. 2004). Mice immunized with tick histamine release factor (tHRF) also showed substantial reduction in spirochete transmission (Dai et al. 2010). Twenty to thirty percent of tHRF-immunized mice were fully protected from spirochete transmission (Dai et al. 2010). Recently, the tick salivary lectin pathway inhibitor (TSLPI) from I. scapularis was shown to prevent killing of B. burgdorferi from host complement system (Schuijt et al. 2011). Mice vaccinated with TSLPI showed $30 \%$ reduction in spirochete loads, but did not completely block transmission (Schuijt et al. 2011). Due to lower percentage of protection of mice seen upon TSLPI immunization, the authors in the study proposed a combination vaccine therapy to prevent Lyme disease (Schuijt et al. 2011). In Northeastern part of the United States, I. scapularis ticks survive overwintering stages (Anderson and Magnarelli 2008). Recently, we identified an antifreeze glycoprotein (IAFGP) molecule in I. scapularis and $I$. ricinus ticks (Neelakanta et al. 2010). IAFGP is found to be critical for the survival of ticks at cold temperatures (Neelakanta et al. 2010). Interestingly, $A$. phagocytophilum-infected ticks produced more IAFGP and showed greater resistance to cold temperatures, suggesting a symbiotic relationship of this microbe with its vector host (Neelakanta et al. 2010). RNA interference of IAFGP showed significant reduction of A. phagocytophilum acquisition in ticks when fed on infected mice (Neelakanta et al. 2010). These results clearly elucidate the important role of IAFGP in ticks. Salp16, a 16-kDa tick salivary gland protein, is shown to be critical for $A$. phagocytophilum survival in ticks (Sukumaran et al. 2006). RNA interference (RNAi) of salp16 expression showed significant $(90 \%)$ reduction of A. phagocytophilum migration from gut to salivary glands (Sukumaran et al. 2006). Our recent study showed that A. phagocytophilum induces tick actin phosphorylation to selectively regulate salp16 gene expression (Sultana et al. 2010). We found substantial increase in G-actin content in tick nuclear extracts upon A. phagocytophilum infection that subsequently influenced salp16 gene expression (Sultana et al. 2010). These results suggest important role of Salp16 in tick-A. phagocytophilum interaction (Sultana et al. 2010). Our study also demonstrated importance of $I$. scapularis p21-activated kinase, PI3-kinase and G-proteins in A. phagocytophilum survival in ticks (Sultana et al. 2010). Study from Labuda et al. (2006) showed that immunization of mice with $64 \mathrm{TPR}$, a tick cement protein protected mice from tick-borne encephalitis virus transmission (Labuda et al. 2006). Viral titers were reduced to more than $50 \%$ in vaccinated mice in comparison to the controls (Labuda et al. 2006). 64TPR is a 15-KDa protein that express at higher levels during tick feeding (Labuda et al. 2006). Moreover, 64TPR has been shown as a broadspectrum vaccine candidate against several tick species (Labuda et al. 2006).

Homologues of Salp15 have been identified in I. pacificus, I. persulcatus and I. sinensis that transmit Borrelia (Table 1) in Western North America and Asia (Hojgaard et al. 2009; Wang et al. 2014b). Three Salp15 homologues were identified in $I$. ricinus, where one of the predicted protein showed $80 \%$ and two other proteins showed $60 \%$ similarity with I. scapularis Salp15 (Hovius et al. 2007). Recent study has identified 5 and 3 Salp15-like proteins in I. persulcatus and I. sinensis ticks, respectively (Wang 
et al. 2014b). Intraspecies and interspecies comparison of these proteins with other Salp15 proteins revealed equal degree of similarity (Wang et al. 2014b). High degree of similarity was evident in the 53-amino acid stretches at the C-terminus of the protein (Hojgaard et al. 2009). These studies have demonstrated that Salp15 and Salp15like proteins are distributed in three clades and represent a multigene family (Hojgaard et al. 2009; Hovius et al. 2007; Wang et al. 2014b). Homologues of Salp16 have been identified in I. persulcatus Schulze ticks (Hidano et al. 2014). These proteins were designated as Salp16 Iper1 and Iper2 (Hidano et al. 2014). I. persulcatus Salp16 proteins have been shown to attenuate oxidative burst of the bovine neutrophils and inhibited migration induced by chemoattractant interleukin-8 (Hidano et al. 2014). This study provides important information on the role of Salp16 in tick blood feeding and as immunosuppressant of neutrophils (Hidano et al. 2014). Homologues of TROSPA have been identified in I. ricinus and I. persulcatus ticks (Konnai et al. 2012). I. persulcatus TROSPA was found to be 88.2 and $87.8 \%$ identical to I. scapularis and I. ricinus TROSPA, respectively (Figlerowicz et al. 2013; Konnai et al. 2012). Figlerowicz et al. (2013) showed that I. ricinus recombinant TROSPA retained capacity of native protein to form a complex with OspA and induced significant level of $\mathrm{IgG}$ in orally immunized animals (Figlerowicz et al. 2013). Recent study has used Anaplasma and Babesia infection model to characterize Subolesin (SUB), SILK and TROSPA as potential antigens to control cattle tick infestations (Antunes et al. 2014). Rabbit polyclonal antibodies generated against SUB, SILK and TROSPA were added to uninfected or infected bovine blood to capillary feed Rhipicephalus microplus ticks (Antunes et al. 2014). Even though results from capillary feeding showed substantial effect on tick weight and oviposition, pathogen levels were unaltered in ticks treated with these antibodies in comparison to the control ticks (Antunes et al. 2014). Due to tick-to-tick variations, this study concluded that capillary feeding was not an ideal technique to characterize efficacy of a vaccine candidate (Antunes et al. 2014). Recent study analyzed whether adaptive evolution has shaped the Salp15 protein family (Schwalie and Schultz 2009). Evidence was seen at several positions within the Salp15 protein family showing phases of positive selection (Schwalie and Schultz 2009). It would be interesting to determine whether the set of several conserved proteins that function in tick-pathogen interactions is also under positive selection. Like Salp15, if Salp16, TROSPA and SUB are also under positive selection, it might be interesting to characterize whether these proteins play differential roles in the interactions with various pathogens (Table 1).
Anti-Haemaphysalis Vaccine Candidates

Some of the Haemaphysalis species transmit pathogens to humans that cause diseases like Lyme borreliosis and spotted fever (Table 1). Study from Chai et al. (2009) has identified 12 unique cDNA sequences in a phage library that encode putative immunodominant antigens in Haemaphysalis longicornis. Harnnoi et al. (2006) have identified genes encoding cement-like antigens in H. longicornis. These cement-like proteins in H. longicornis were upregulated upon feeding (Harnnoi et al. 2006). HISP, a cubilin-related serine proteinase was identified in $H$. longicornis (Miyoshi et al. 2004). HISP expression was highly upregulated during tick feeding indicating its role in digestion of host blood components (Miyoshi et al. 2004). Recent study characterized acid phosphatase from $H$. longicornis (Zhang et al. 2011). Rabbits vaccinated with recombinant acid phosphatase conferred protective immunity, resulting in $28 \%$ mortality and $10.6 \%$ reduction in body weight of adult ticks (Zhang et al. 2011). Scavenger receptors are predominantly expressed by mammalian macrophages and are shown to be important in innate immunity (Aung et al. 2011). Study from Aung et al. (2011) have identified Scavenger receptor homologue (HISRB) in H. longicornis for the first time in Chelicerata that include ticks, horseshoe crabs, scorpions, spiders and mites. Silencing of HISRB gene resulted in reduced tick body weight (Aung et al. 2011). It is not surprising to hypothesize that HISRB can be considered as an effective candidate for the development of vaccine against $H$. longicornis (Aung et al. 2011). Follistatin-related protein (Zhou et al. 2006a) and Valosin-containing protein (Boldbaatar et al. 2007) have been identified in H. longicornis that are critical for tick oviposition and engorgement. A salivary gland protein Longistatin, a serine protease with two EF-domains has been recently identified in H. longicornis (Anisuzzaman et al. 2012). The study showed that Longistatin hydrolyzed fibrinogen and activated plasminogen into its active form plasmin (Anisuzzaman et al. 2012). Immunization of Longistatin in animals produced high IgG titers against ticks that revealed $54 \%$ tick repletion, and reduction of tick body weight to $11 \%$ in comparison to controls (Anisuzzaman et al. 2012). Zhou et al. (2006b) cloned and characterized Cystatin gene from $H$. longicornis. Their study revealed that recombinant Cystatin protein showed growth-inhibitory effect on Babesia bovis cultured in vitro. The authors indicated that Cystatin is critical for tick immunity (Zhou et al. 2006b).

Anti-Dermacentor Vaccine Candidates

Several species of Dermacentor ticks are known carriers of many human pathogens (Table 1). Subolesin, an ortholog 
of insect akirins is a highly conserved protein that could be potentially considered as vaccine against various blood sucking vectors including ticks and mosquitoes (de la Fuente et al. 2011). Hu et al. (2014) have cloned, characterized and analyzed immunogenicity of $D$. silvarum SUB. Their analysis showed that anti-D. silvarum serum recognized recombinant SUB, suggesting an host immune response against this protein ( $\mathrm{Hu}$ et al. 2014). Fibrinogenrelated proteins are shown to be important for tick innate system and are suggested to be potential candidates for the development of anti-tick vaccines (Hajdusek et al. 2013). Recent study has identified fibrinogen-related proteins from D. marginatus, Rhipicephalus appendiculatus, R. pulchellus and R. sanguineus (Sterba et al. 2011). Dermacentor ticks feed for 6-10 days with varying expression levels of several tick proteins involved in the process of blood feeding (Sonenshine and Roe 2014). Salivary gland extracts isolated at different times during D. andersoni feeding showed esterase activities (Gordon and Allen 1987). Another study determined immunogenic proteins in $R$. sanguineus and D. reticulatus ticks using 2-D gel electrophoresis (Vu Hai et al. 2013). IgG response against bite of $R$. sanguineus and $D$. reticulatus ticks were determined in rabbits ( $\mathrm{Vu} \mathrm{Hai}$ et al. 2013). Using antiserum from rabbits exposed to these two ticks, important candidate antigens for the development of anti-tick vaccines were identified (Vu Hai et al. 2013). These include but are not limited to heme-binding, cement and Vitellogenin-2 proteins from $R$. sanguineus and hemelipoglycoprotein precursor, Serpin-2 precursor, Calreticulin from $D$. reticulatus (Vu Hai et al. 2013). The major hemelipoglycocarrier protein $(\mathrm{CP})$ was identified from $D$. variabilis (Donohue et al. 2008). Expression and domain structure of CP matched Vitellogenin that suggested its role in heme sequestration, evolution of hematophagy and host complementation (Donohue et al. 2008). Anderson et al. (2008) explored D. variabilis mialome of ticks and identified 82 transcripts that encode proteins putatively associated in blood meal digestion, enzymes involved in oxidative stress, peptidase inhibitors, protein digestion, protein and lipid binding, mucins and iron/heme metabolism. Transcripts encoding lectin- and allergen-like proteins were also identified in that screen (Anderson et al. 2008). Studies like these would provide evidences on the role of several proteins in blood digestion, antimicrobial activity and transmission of pathogens that could be envisioned for the development of anti-Dermacentor vaccines.

\section{Anti-Amblyomma Vaccine Candidates}

Amblyomma ticks are vectors for several Rickettsia spp. and Francisella tularensis that cause diseases in humans (Table 1). Mulenga et al. (2013b) have identified tick feeding stimuli responsive gene AamAV422 gene that belong to a novel group of arthropod proteins. AamAV422 contain 14 cysteine amino acid residues that are predicted to form seven disulfide bonds (Mulenga et al. 2013b). AamAV422 has been suggested to mediate tick antihemostasis and anti-complement activity (Mulenga et al. 2013b). RNA interference against AamAV422 caused significant $(44 \%)$ reduction in tick engorgement weights (Mulenga et al. 2013b). This study concluded that AamAV422 could be considered as a potential candidate for a cocktail or multivalent tick vaccine (Mulenga et al. 2013b). A. americanum saliva cysteine proteases have been shown to delay plasma clotting and inhibit platelet aggregation (Mulenga et al. 2013a). Three of the insulin-like growth factor binding proteins (IGFBP) has been identified in $A$. americanum (Mulenga and Khumthong 2010b). Silencing of the IGFBPs had substantial effect on tick feeding (Mulenga and Khumthong 2010b). Organic anion $\mathrm{Na}^{+}$independent transporting polypeptide (AamOatp) that function in wide range of endogenous and xenobiotic compounds transport across plasma membrane has been identified in A. americanum (Mulenga et al. 2008). Silencing of AamOatp showed significant reduction in blood intake by ticks (Mulenga et al. 2008). Recently tick CD147 receptor was identified and characterized in $A$. americanum (Mulenga and Khumthong 2010a). CD147 receptors are cell-surface glycoprotein in IgG family that play an important role in extracellular matrix remodeling (Huet et al. 2008). The expression of A. americanum CD147 receptor was found to be up regulated upon tick feeding in salivary glands, but remain unaltered in gut suggesting differential roles in these tissues (Mulenga and Khumthong 2010a). The authors of this research study showed that CD147 receptor silencing abrogates tick intermolt growth (Mulenga and Khumthong 2010a). Study from de la Fuente et al. (2010) have used four cDNA clones encoding putative threonyl-tRNA synthetase (2C9), 60S ribosomal proteins L13a (2D10) and L13e (2B7) and interphase cytoplasm foci protein 45 (2G7) for immunization studies in cattle. Immunization with $2 \mathrm{G} 7$ showed significant $(E>55 \%)$ control of adult ticks (de la Fuente et al. 2010). Immunization with 2D10, 2G7 and subolesin affected control of both nymphs and adults (de la Fuente et al. 2010).

\section{Anti-Rhipicephalus Vaccine Candidates}

Rhipicephalus ticks transmit pathogens that cause diseases in humans (Table 1). Study from Rodriguez-Mallon focused on Rhipicephalus ribosomal proteins (RodriguezMallon et al. 2012) identified a unique immunogenic region of protein $\mathrm{P} 0$. This protein is important in the assembly of 60S ribosomal subunit (Rodriguez-Mallon et al. 2012). The 
immunogenic region of Rhipicephalus species is not conserved in comparison to host P0 protein (Rodriguez-Mallon et al. 2012). Animals were challenged with peptide generated based on the sequence from non-conserved region (Rodriguez-Mallon et al. 2012). The results showed that when larvae were fed on vaccinated animals, significant reduction of viability of newly molted $R$. sanguineus nymphs were noted (Rodriguez-Mallon et al. 2012). More than $90 \%$ reduction was observed in tick egg hatching (Rodriguez-Mallon et al. 2012). Overall, their study provided evidence that vaccination of animals with P0 significantly reduced survival of ticks. $R$. microplus gut cells express Bm86, a membrane-bound glycoprotein on the surface (De La Fuente et al. 2000; Willadsen et al. 1989; Willadsen and Jongejan 1999). Even though the unknown biological function of Bm86 in ticks is yet to be explored, several studies have used Bm86 as a protective antigen against protection from infestation of cattle ticks $R$. microplus and $R$. annulatus (Carreon et al. 2012; de la Fuente et al. 1999; De La Fuente et al. 2000; RodriguezMallon et al. 2012; Rodriguez-Valle et al. 2012; Willadsen and Jongejan 1999; Willadsen et al. 1989, 1996). Protection of Cattle from $R$. microplus infestations by $\mathrm{Bm} 86$ vaccination was achieved due to the generation of antigenspecific antibodies by the host that subsequently affected tick infestations and fertility (Willadsen and Jongejan 1999; Willadsen et al. 1989, 1996). Bm86-based TickGARD and Gavac vaccines are now commercially available anti-vector vaccines for animals (Odongo et al. 2007; Valle et al. 2004; Willadsen et al. 1995). Perez-Perez and co-authors vaccinated domestic dogs with Bm86 and infested them with three instars of $R$. sanguineus (PerezPerez et al. 2010). Their study revealed that ticks fed on vaccinated animals had reduced viability in comparison to controls. $R$. sanguineus was used to evaluate silencing effects of tick protective antigens 4D8 and Rs86, homologues of Bm86 (de la Fuente et al. 2006). Silencing of 4D8 alone had effect on tick feeding, attachment and oviposition (de la Fuente et al. 2006). Silencing of Rs86 had an effect on tick weight and oviposition (de la Fuente et al. 2006). Combined silencing of expression of both genes (4D8 and Rs86) had significant effect on $R$. sanguineus survival, attachment, feeding, weight and oviposition (de la Fuente et al. 2006). The authors from this study proposed the development of multi-antigenic vaccines to prevent infestation from $R$. sanguineus (de la Fuente et al. 2006). This type of multi-antigenic vaccine comprising Vitellindegrading cysteine endopeptidase and Boophilus yolk procathepsin both from $R$. microplus and glutathione S-transferase from $H$. longicornis was evaluated against the cattle tick R. microplus (Parizi et al. 2012). Increased antibody titers were evident in vaccinated animals (Parizi et al. 2012). The multi-antigenic vaccine administration in animals had substantial effect on tick infestations, where significantly lower numbers of semi-engorged female ticks were noted (Parizi et al. 2012). Vaccination with truncated construct of cement protein (64TRPs) derived from $R$. appendiculatus showed significant cross protection against $R$. sanguineus (Trimnell et al. 2005). Recently, three major glycoproteins (GLPs) of sizes about 90, 66 and $40 \mathrm{kDa}$ have been purified from adult and larval Hyalomma dromedarii (camel ticks) (El Hakim et al. 2011). Immunization with GLPs protected animals from $H$. dromedarii infestations (El Hakim et al. 2011). Immune sera raised against $H$. dromedarii GLPs were able to recognize proteins from $R$. sanguineus, suggesting its efficacy as a potent vaccine candidate against other ticks species (El Hakim et al. 2011). Sialotranscriptome of $R$. sanguineus has now been determined that could aid in the additional discovery of candidate molecules important for the development of anti-Rhipicephalus vaccines (Anatriello et al. 2010).

\section{Anti-Ornithodoros Vaccine Candidates}

Ornithodoros tick species are important vectors for the transmission of relapsing fever spirochetes (Table 1). Astigarraga et al. (1995) performed a study by immunizing animals with concealed antigens or salivary gland extracts from $O$. erraticus. While immunization with concealed antigens did not result in any protection against ticks, immunization with salivary gland extracts significantly reduced blood ingestion (40-60\%) and fecundity (40-60 \%) in ticks (Astigarraga et al. 1995). This was also evident in immunization with salivary gland extracts prepared from O. moubata (Astigarraga et al. 1995). Factor Xa inhibitor (fXaI; anti-coagulation factor) has been identified in O. savignyi that shows $46 \%$ identity and $78 \%$ similarity in amino acid sequence with fXaI from $O$. moubata (Joubert et al. 1998). Midgut membrane extracts prepared from $O$. erraticus were administered to mice and pigs to test protective responses in these animals against ticks (Manzano-Roman et al. 2006). When midgut extracts were injected with Freund's adjuvants into these animals, $80 \%$ of the immature forms of the ticks were killed in the first $72 \mathrm{~h}$ post-feeding and also decreased fecundity in females by more than $50 \%$ (Manzano-Roman et al. 2006). The significant rate of killing was attributed to the damage of midgut wall of ticks (Manzano-Roman et al. 2006). It was also noted that some proteins present in the midgut extracts were expressed in all developmental stages of $O$. erraticus ticks and found to be induced upon feeding suggesting important targets for the vaccine development (ManzanoRoman et al. 2006). Subolesin orthologs were identified in $O$. erraticus and $O$. moubata ticks (Manzano-Roman et al. $2012 b)$. The Subolesin of both these ticks show more than 69 and $74 \%$ identify with Subolesin from hard ticks in 
their nucleotide and amino acid sequences, respectively (Manzano-Roman et al. 2012b). RNAi-mediated silencing of Subolesin inhibited Ornithodoros oviposition indicating importance of this protein in tick oviposition (ManzanoRoman et al. 2012b). Immunization studies with soft tick recombinant Subolesin induced partial protective effect resulting in reduced oviposition rates of these ticks (Manzano-Roman et al. 2012b). A lectin, designated as Dorin $\mathrm{M}$, with high hemagglutinating activity was isolated from O. moubata (Kovar et al. 2000). This was a first report of purified lectin from ticks (Kovar et al. 2000). Significant levels of enolase have been found in the saliva of $O$. moubata ticks (Diaz-Martin et al. 2013). Enolase is a glycolytic enzyme that acts as plasminogen receptor on cell surface to promote fibrinolysis and extracellular matrix degradation (Diaz-Martin et al. 2013). The function of enolase could be important for ticks to digest clots and also to prevent clotting formed during feeding (Diaz-Martin et al. 2013). RNAi and immunization studies revealed that O. moubata enolase could be involved in tick reproduction (Diaz-Martin et al. 2013). Using self-assembled protein arrays, a recent study has identified important tick molecules in the O. moubata salivary gland expression library (Manzano-Roman et al. 2012a). Several clones that bind to human P-selectin were identified in the screen (ManzanoRoman et al. 2012a). Secreted tick phospholipase A2, which could function as potential new ligand for P-selectin was also identified (Manzano-Roman et al. 2012a). This strategy provides one of the important breakthroughs in the identification of several vaccine candidates in ticks (Manzano-Roman et al. 2012a). Cystatin from O. moubata has been identified (Salat et al. 2010). Immunization studies with recombinant Cystatin (OmC2) significantly suppressed survival of $O$. moubata (Salat et al. 2010). A tick salivary gland glycoprotein of $44 \mathrm{kDa}(\mathrm{Om} 44)$ that carries heparin determinant has been identified in $O$. moubata salivary gland extracts (Garcia-Varas et al. 2010). Partial characterization of this protein revealed that $\mathrm{Om} 44$ binds host P-selectin, that presumably prevents the adhesion of leucocytes and platelets to vessel walls (GarciaVaras et al. 2010). Om44 cannot be recognized by host immune system, but can be neutralized by antibodies. This study found significant inhibition of $O$. moubata feeding on vaccinated animals in comparison to the controls, making new prospects in the development of anti-O. moubata and perhaps other Ornithodoros species vaccine (Garcia-Varas et al. 2010).

\section{Conclusions}

In this review, we provide an overview of potential candidates for the development of anti-vector vaccines against human diseases transmitted by various hard and soft ticks. Several commercial and technical challenges need to be overcome in order to develop an effective anti-vector vaccine for human use (de la Fuente et al. 2007; Merino et al. 2013). Some of the challenges to overcome are: (1) limitation in the molecular evidences to understand how anti-vector vaccine works in humans; (2) overall ineffectiveness of anti-vector vaccines to control certain tick population (De La Fuente et al. 2000; de Vos et al. 2001); (3) limited interest for commercialization and vaccine production on large scale and (4) insufficient knowledge dispersal to the public about the use of anti-vector vaccines.

Over the past decade, several studies have used genomics, transcriptomics and proteomics approaches to identify some of the potential candidates for the development of anti-vector vaccines (Dinglasan et al. 2005; Geiger et al. 2011; Gibson et al. 2013; Heekin et al. 2013a, b; Hill et al. 2005; Patramool et al. 2012; Ramabu et al. 2010; Schwarz et al. 2013; Valenzuela 2004b; Vernick and Waters 2004; Villar et al. 2014a, b). With the availability of already abundant amount of information on several tick genomes, transcriptomes and proteomes, future studies need to focus on developing suitable animal models and approaches to evaluate proper efficacy of a molecule as a vaccine candidate for human trials. Studies using multidisciplinary approaches such as use of bioinformatic analysis for epitope prediction, protein folding and posttranslational modifications on a vaccine candidate molecule would strengthen development of anti-vector vaccines. In addition, development of multivalent-vaccine formulations comprising vaccines targeting both vector and pathogen would provide a significant leap in this research area to move forward from bench-to-bedside.

Acknowledgments This work was supported in part by National Institute of Health grants AI090197 (GN) and AI092156 (HS) and independent Start-Up funds from Old Dominion University to GN and HS.

Conflict of interest Authors declare no conflicts of interest.

Open Access This article is distributed under the terms of the Creative Commons Attribution License which permits any use, distribution, and reproduction in any medium, provided the original author(s) and the source are credited.

\section{References}

Anatriello E, Ribeiro JM, de Miranda-Santos IK et al (2010) An insight into the sialotranscriptome of the brown dog tick Rhipicephalus sanguineus. BMC Genomics 11:450

Anderson JF, Magnarelli LA (2008) Biology of ticks. Infect Dis Clin North Am 22:195-215

Anderson JM, Sonenshine DE, Valenzuela JG (2008) Exploring the mialome of ticks: an annotated catalogue of midgut transcripts 
from the hard tick, Dermacentor variabilis (Acari: Ixodidae). BMC Genom 9:552

Anisuzzaman Islam MK, Alim MA et al (2012) Longistatin is an unconventional serine protease and induces protective immunity against tick infestation. Mol Biochem Parasitol 182:45-53

Antunes S, Merino O, Mosqueda J et al (2014) Tick capillary feeding for the study of proteins involved in tick-pathogen interactions as potential antigens for the control of tick infestation and pathogen infection. Parasit Vectors 7:42

Astigarraga A, Oleaga-Perez A, Perez-Sanchez R et al (1995) A study of the vaccinal value of various extracts of concealed antigens and salivary gland extracts against Ornithodoros erraticus and Ornithodoros moubata. Vet Parasitol 60:133-147

Aung KM, Boldbaatar D, Liao M et al (2011) Identification and characterization of class B scavenger receptor CD36 from the hard tick, Haemaphysalis longicornis. Parasitol Res 108:273-285

Barry EM, Cole LE, Santiago AE (2009) Vaccines against tularemia. Hum Vaccin 5:832-838

Billingsley PF, Foy B, Rasgon JL (2008) Mosquitocidal vaccines: a neglected addition to malaria and dengue control strategies. Trends Parasitol 24:396-400

Boldbaatar D, Battsetseg B, Hatta T et al (2007) Valosin-containing protein from the hard tick Haemaphysalis longicornis: effects of dsRNA-mediated HIVCP gene silencing. Biochem Cell Biol 85:384-394

Callow LL (1978) Ticks and tick-borne diseases as a barrier to the introduction of exotic cattle to the tropics. Wld Anim Rev 28:20-25

Carreon D, de la Lastra JM, Almazán C et al (2012) Vaccination with BM86, subolesin and akirin protective antigens for the control of tick infestations in white tailed deer and red deer. Vaccine 30:273-279

CDC (2014) Tickborne Diseases of the United states Centers for Disease Control and Prevention, US Department of Health and Human Services a Reference Manual for Health Care Providers, pp 1-21

Chai HPLG, Zhang L, Gong ZL et al (2009) Construction of cDNA expression library of unfed female Haemaphysalis longicornis and immuno-screening. Zhongguo Ji Sheng Chong Xue Yu Ji Sheng Chong Bing Za Zhi 27:6-10

Conlan JW (2011) Tularemia vaccines: recent developments and remaining hurdles. Future Microbiol 6:391-405

Coutinho-Abreu IV, Ramalho-Ortigao M (2010) Transmission blocking vaccines to control insect-borne diseases: a review. Mem Inst Oswaldo Cruz 105:1-12

Coutinho-Abreu IV, Zhu KY, Ramalho-Ortigao M (2009) Transgenesis and paratransgenesis to control insect-borne diseases: current status and future challenges. Parasitol Int 59:1-8

Dai J, Wang P, Adusumilli S et al (2009) Antibodies against a tick protein, Salp15, protect mice from the Lyme disease agent. Cell Host Microbe 6:482-492

Dai J, Narasimhan S, Zhang L et al (2010) Tick histamine release factor is critical for Ixodes scapularis engorgement and transmission of the lyme disease agent. PLoS Pathog 6:e1001205

de la Fuente J, Merino O (2013) Vaccinomics, the new road to tick vaccines. Vaccine 31:5923-5929

de la Fuente J, Rodríguez M, Montero C et al (1999) Vaccination against ticks (Boophilus spp.): the experience with the Bm86based vaccine. Gavac Genet Anal 15:143-148

De La Fuente J, Rodriguez M, Garcia-Garcia JC (2000) Immunological control of ticks through vaccination with Boophilus microplus gut antigens. Ann NY Acad Sci 916:617-621

de la Fuente J, Almazan C, Naranjo V et al (2006) Synergistic effect of silencing the expression of tick protective antigens 4D8 and Rs86 in Rhipicephalus sanguineus by RNA interference. Parasitol Res 99:108-113 de la Fuente J, Almazan C, Canales M et al (2007) A ten-year review of commercial vaccine performance for control of tick infestations on cattle. Anim Health Res Rev 8:23-28

de la Fuente J, Manzano-Roman R, Naranjo V et al (2010) Identification of protective antigens by RNA interference for control of the lone star tick, Amblyomma americanum. Vaccine 28:1786-1795

de la Fuente J, Moreno-Cid JA, Canales M et al (2011) Targeting arthropod subolesin/akirin for the development of a universal vaccine for control of vector infestations and pathogen transmission. Vet Parasitol 181:17-22

de Vos S, Zeinstra L, Taoufik O et al (2001) Evidence for the utility of the Bm86 antigen from Boophilus microplus in vaccination against other tick species. Exp Appl Acarol 25:245-261

Demma LJ, Eremeeva M, Nicholson WL et al (2006) An outbreak of Rocky Mountain Spotted Fever associated with a novel tick vector, Rhipicephalus sanguineus, in Arizona, 2004: preliminary report. Ann NY Acad Sci 1078:342-343

Desenclos JC (2011) Transmission parameters of vector-borne infections. Med Mal Infect 41:588-593

Diaz-Martin V, Manzano-Roman R, Oleaga A et al (2013) Cloning and characterization of a plasminogen-binding enolase from the saliva of the argasid tick Ornithodoros moubata. Vet Parasitol 191:301-314

Dinglasan RR, Valenzuela JG, Azad AF (2005) Sugar epitopes as potential universal disease transmission blocking targets. Insect Biochem Mol Biol 35:1-10

Donohue KV, Khalil SM, Mitchell RD et al (2008) Molecular characterization of the major hemelipoglycoprotein in ixodid ticks. Insect Mol Biol 17:197-208

El Hakim AE, Shahein YE, Abdel-Shafy S et al (2011) Evaluation of glycoproteins purified from adult and larval camel ticks (Hyalomma dromedarii) as a candidate vaccine. J Vet Sci 12:243-249

Figlerowicz M, Urbanowicz A, Lewandowski D et al (2013) Functional insights into recombinant TROSPA protein from Ixodes ricinus. PLoS One 8:e76848

Garcia-Varas S, Manzano-Roman R, Fernandez-Soto P et al (2010) Purification and characterisation of a P-selectin-binding molecule from the salivary glands of Ornithodoros moubata that induces protective anti-tick immune responses in pigs. Int $\mathrm{J}$ Parasitol 40:313-326

Gardner CL, Ryman KD (2010) Yellow fever: a reemerging threat. Clin Lab Med 30:237-260

Geiger A, Simo G, Grebaut P et al (2011) Transcriptomics and proteomics in human African trypanosomiasis: current status and perspectives. J Proteomics 74:1625-1643

Gibson AK, Smith Z, Fuqua C et al (2013) Why so many unknown genes? Partitioning orphans from a representative transcriptome of the lone star tick Amblyomma americanum. BMC Genom 14:135

Githeko AK, Lindsay SW, Confalonieri UE et al (2000) Climate change and vector-borne diseases: a regional analysis. Bull World Health Organ 78:1136-1147

Goddard J (2008) Dynamics of arthropod-borne diseases infectious diseases and arthropods. Springer XVI, 131 illus., Hardover, p 251

Gordon JR, Allen JR (1987) Isolation and characterization of salivary antigens from the female tick, Dermacentor andersoni. Parasite Immunol 9:337-352

Hajdusek O, Sima R, Ayllon N et al (2013) Interaction of the tick immune system with transmitted pathogens. Front Cell Infect Microbiol 3:26

Harnnoi T, Sakaguchi T, Xuan X et al (2006) Identification of genes encoding cement-like antigens expressed in the salivary glands of Haemaphysalis longicornis. J Vet Med Sci 68:1155-1160 
Heekin AM, Guerrero FD, Bendele KG et al (2013a) Gut transcriptome of replete adult female cattle ticks, Rhipicephalus (Boophilus) microplus, feeding upon a Babesia bovis-infected bovine host. Parasitol Res 112:3075-3090

Heekin AM, Guerrero FD, Bendele KG et al (2013b) The ovarian transcriptome of the cattle tick, Rhipicephalus (Boophilus) microplus, feeding upon a bovine host infected with Babesia bovis. Parasit Vectors 6:276

Heinz FX, Stiasny K (2012) Flaviviruses and flavivirus vaccines. Vaccine 30:4301-4306

Hidano A, Konnai S, Yamada S et al (2014) Suppressive effects of neutrophil by Salp16-like salivary gland proteins from Ixodes persulcatus Schulze tick. Insect Mol Biol 23:466-474

Hill CA, Kafatos FC, Stansfield SK et al (2005) Arthropod-borne diseases: vector control in the genomics era. Nat Rev Microbiol 3:262-268

Hojgaard A, Biketov SF, Shtannikov AV et al (2009) Molecular identification of Salp15, a key salivary gland protein in the transmission of lyme disease spirochetes, from Ixodes persulcatus and Ixodes pacificus (Acari: Ixodidae). J Med Entomol 46:1458-1463

Hovius JW, Ramamoorthi N, Van't Veer C et al (2007) Identification of Salp15 homologues in Ixodes ricinus ticks. Vector Borne Zoonotic Dis 7:296-303

Hu Y, Zeng H, Zhang J et al (2014) Gene cloning, expression and immunogenicity of the protective antigen subolesin in Dermacentor silvarum. Korean J Parasitol 52:93-97

Huet E, Gabison EE, Mourah S et al (2008) Role of emmprin/CD147 in tissue remodeling. Connect Tissue Res 49:175-179

Joubert AM, Louw AI, Joubert F et al (1998) Cloning, nucleotide sequence and expression of the gene encoding factor $\mathrm{Xa}$ inhibitor from the salivary glands of the tick, Ornithodoros savignyi. Exp Appl Acarol 22:603-619

Juncadella IJ, Anguita J (2009) The immunosuppressive tick salivary protein, Salp15. Adv Exp Med Biol 666:121-131

Konnai S, Yamada S, Imamura S et al (2012) Identification of TROSPA homologue in Ixodes persulcatus Schulze, the specific vector for human Lyme borreliosis in Japan. Ticks Tick Borne Dis 3:75-77

Kovar V, Kopacek P, Grubhoffer L (2000) Isolation and characterization of Dorin M, a lectin from plasma of the soft tick Ornithodoros moubata. Insect Biochem Mol Biol 30:195-205

Labuda M, Trimnell AR, Licková M et al (2006) An antivector vaccine protects against a lethal vector-borne pathogen. PLoS Pathog 2:e27

Malkin EM, Durbin AP, Diemert DJ et al (2005) Phase 1 vaccine trial of Pvs25H: a transmission blocking vaccine for Plasmodium vivax malaria. Vaccine 23:3131-3138

Maltezou HC, Andonova R, Andraghetti R et al (2010) CrimeanCongo hemorrhagic fever in Europe: current situation calls for preparedness. Eurosurveillance 15:1-4

Manzano-Roman R, Encinas-Grandes A, Perez-Sanchez R (2006) Antigens from the midgut membranes of Ornithodoros erraticus induce lethal anti-tick immune responses in pigs and mice. Vet Parasitol 135:65-79

Manzano-Roman R, Díaz-Martín V, González-González M et al (2012a) Self-assembled protein arrays from an Ornithodoros moubata salivary gland expression library. J Proteome Res 11:5972-5982

Manzano-Roman R, Diaz-Martin V, Oleaga A et al (2012b) Subolesin/akirin orthologs from Ornithodoros spp. soft ticks: cloning, RNAi gene silencing and protective effect of the recombinant proteins. Vet Parasitol 185:248-259

Merino O, Alberdi P, Perez de la Lastra JM et al (2013) Tick vaccines and the control of tick-borne pathogens. Front Cell Infect Microbiol 3:30
Miyoshi T, Tsuji N, Islam MK et al (2004) Cloning and molecular characterization of a cubilin-related serine proteinase from the hard tick Haemaphysalis longicornis. Insect Biochem Mol Biol 34:799-808

Mulenga A, Khumthong R (2010a) Disrupting the Amblyomma americanum (L.) CD147 receptor homolog prevents ticks from feeding to repletion and blocks spontaneous detachment of ticks from their host. Insect Biochem Mol Biol 40:524-532

Mulenga A, Khumthong R (2010b) Silencing of three Amblyomma americanum (L.) insulin-like growth factor binding proteinrelated proteins prevents ticks from feeding to repletion. J Exp Biol 213:1153-1161

Mulenga A, Khumthong R, Chalaire KC et al (2008) Molecular and biological characterization of the Amblyomma americanum organic anion transporter polypeptide. J Exp Biol 211:3401-3408

Mulenga A, Kim T, Ibelli AM (2013a) Amblyomma americanum tick saliva serine protease inhibitor 6 is a cross-class inhibitor of serine proteases and papain-like cysteine proteases that delays plasma clotting and inhibits platelet aggregation. Insect Mol Biol 22:306-319

Mulenga A, Kim TK, Ibelli AM (2013b) Deorphanization and target validation of cross-tick species conserved novel Amblyomma americanum tick saliva protein. Int J Parasitol 43:439-451

Narasimhan S, Sukumaran B, Bozdogan U et al (2007) A tick antioxidant facilitates the Lyme disease agent's successful migration from the mammalian host to the arthropod vector. Cell Host Microbe 2:7-18

Neelakanta G, Sultana H, Fish D et al (2010) Anaplasma phagocytophilum induces Ixodes scapularis ticks to express an antifreeze glycoprotein gene that enhances their survival in the cold. J Clin Invest 120:3179-3190

Ødegaard F (2000) How many species of arthropods? Erwin's estimate revised. Biol J Linnean Society 71:583-597

Odongo D, Kamau L, Skilton R et al (2007) Vaccination of cattle with TickGARD induces cross-reactive antibodies binding to conserved linear peptides of $\mathrm{Bm} 86$ homologues in Boophilus decoloratus. Vaccine 25:1287-1296

Oliveira F, Jochim RC, Valenzuela JG et al (2009) Sand flies, Leishmania, and transcriptome-borne solutions. Parasitol Int 58:1-5

Orlinger KK, Hofmeister Y, Fritz R et al (2011) A tick-borne encephalitis virus vaccine based on the European prototype strain induces broadly reactive cross-neutralizing antibodies in humans. J Infect Dis 203:1556-1564

Pal U, Li X, Wang T et al (2004) TROSPA, an Ixodes scapularis receptor for Borrelia burgdorferi. Cell 119:457-468

Parizi LF, Reck J Jr, Oldiges DP et al (2012) Multi-antigenic vaccine against the cattle tick Rhipicephalus (Boophilus) microplus: a field evaluation. Vaccine 30:6912-6917

Patramool S, Choumet V, Surasombatpattana P et al (2012) Update on the proteomics of major arthropod vectors of human and animal pathogens. Proteomics 12:3510-3523

Pattnaik P (2006) Kyasanur forest disease: an epidemiological view in India. Rev Med Virol 16:151-165

Perez-Perez D, Bechara GH, Machado RZ et al (2010) Efficacy of the Bm86 antigen against immature instars and adults of the dog tick Rhipicephalus sanguineus (Latreille, 1806) (Acari: Ixodidae). Vet Parasitol 167:321-326

Ramabu SS, Ueti MW, Brayton KA et al (2010) Identification of Anaplasma marginale proteins specifically upregulated during colonization of the tick vector. Infect Immun 78:3047-3052

Ramamoorthi N, Narasimhan S, Pal U et al (2005) The Lyme disease agent exploits a tick protein to infect the mammalian host. Nature 436:573-577

Randolph SE (2008) Tick-borne encephalitis incidence in Central and Eastern Europe: consequences of political transition. Microbes Infect 10:209-216 
Reed DS, Smith LP, Cole KS et al (2014) Live attenuated mutants of Francisella tularensis protect rabbits against aerosol challenge with a virulent type A strain. Infect Immun 82:2098-2105

Rodriguez-Mallon A, Fernández E, Encinosa PE et al (2012) A novel tick antigen shows high vaccine efficacy against the dog tick, Rhipicephalus sanguineus. Vaccine 30:1782-1789

Rodriguez-Valle M, Taoufik A, Valdés M et al (2012) Efficacy of Rhipicephalus (Boophilus) microplus Bm86 against Hyalomma dromedarii and Amblyomma cajennense tick infestations in camels and cattle. Vaccine 30:3453-3458

Salat J, Paesen GC, Rezácová P et al (2010) Crystal structure and functional characterization of an immunomodulatory salivary cystatin from the soft tick Ornithodoros moubata. Biochem J 429:103-112

Saul A, Hensmann M, Sattabongkot J et al (2007) Immunogenicity in rhesus of the Plasmodium vivax mosquito stage antigen Pvs25H with Alhydrogel and Montanide ISA 720. Parasite Immunol 29:525-533

Schuijt TJ, Coumou J, Narasimhan S et al (2011) A tick mannosebinding lectin inhibitor interferes with the vertebrate complement cascade to enhance transmission of the lyme disease agent. Cell Host Microbe 10:136-146

Schwalie PC, Schultz J (2009) Positive selection in tick saliva proteins of the Salp15 family. J Mol Evolution 68:186-191

Schwarz A, von Reumont BM, Erhart J et al (2013) De novo Ixodes ricinus salivary gland transcriptome analysis using two nextgeneration sequencing methodologies. FASEB J 27:4745-4756

Sonenshine DE, Roe R (2014) Biology of ticks, 2nd edn. Oxford University Press, Oxford, $\mathrm{p} 2$

Sterba J, Dupejova J, Fiser M et al (2011) Fibrinogen-related proteins in ixodid ticks. Parasit Vectors 4:127

Sukumaran B, Narasimhan S, Anderson JF et al (2006) An Ixodes scapularis protein required for survival of Anaplasma phagocytophilum in tick salivary glands. J Exp Med 203:1507-1517

Sultana H, Foellmer HG, Neelakanta G et al (2009) Fusion loop peptide of the West Nile virus envelope protein is essential for pathogenesis and is recognized by a therapeutic cross-reactive human monoclonal antibody. J Immunol 183:650-660

Sultana H, Neelakanta G, Kantor FS et al (2010) Anaplasma phagocytophilum induces actin phosphorylation to selectively regulate gene transcription in Ixodes scapularis ticks. J Exp Med 207:1727-1743

Thomas MB, Read AF (2007) Can fungal biopesticides control malaria? Nat Rev Microbiol 5:377-383

Trimnell AR, Davies GM, Lissina O et al (2005) A cross-reactive tick cement antigen is a candidate broad-spectrum tick vaccine. Vaccine 23:4329-4341

Valenzuela JG (2004a) Blood-feeding arthropod salivary glands and saliva. In: Marquardt WC (ed) Biology of disease vectors. Elsevier Academic Press, Burlington, pp 377-386

Valenzuela JG (2004b) Exploring tick saliva: from biochemistry to 'sialomes' and functional genomics. Parasitology 129(Suppl):S83-S94

Valle MR, Mèndez L, Valdez M et al (2004) Integrated control of Boophilus microplus ticks in Cuba based on vaccination with the anti-tick vaccine Gavac. Exp Appl Acarol 34:375-382
Vernick KD, Waters AP (2004) Genomics and malaria control. New Engl J Med 351:1901-1904

Villar M, Popara M, Ayllón N et al (2014a) A systems biology approach to the characterization of stress response in Dermacentor reticulatus tick unfed larvae. PLoS One 9:e89564

Villar M, Popara M, Mangold AJ et al (2014b) Comparative proteomics for the characterization of the most relevant Amblyomma tick species as vectors of zoonotic pathogens worldwide. J Proteomics 105:204-216

$\mathrm{Vu}$ Hai V, Almeras L, Audebert S et al (2013) Identification of salivary antigenic markers discriminating host exposition between two European ticks: Rhipicephalus sanguineus and Dermacentor reticulatus. Comp Immunol Microbiol Infect Dis 36:39-53

Wang HJ, Li XF, Ye Q et al (2014a) Recombinant chimeric Japanese encephalitis virus/tick-borne encephalitis virus is attenuated and protective in mice. Vaccine 32:949-956

Wang X, Huang Y, Niu SB et al (2014b) Genetic Diversity of Salp15 in the Ixodes ricinus Complex (Acari: Ixodidae). PLoS One 9:e94131

WHO (2004) The world health report 2004-changing history. World Health Organization, Geneva

WHO (2013) World malaria report 2013. World Health Organization, Geneva, p 284

WHO (2014) A global brief on vector-borne diseases. World Health Organization, Geneva

Willadsen P, Jongejan F (1999) Immunology of the tick-host interaction and the control of ticks and tick-borne diseases. Parasitol Today 15:258-262

Willadsen P, Riding GA, McKenna RV et al (1989) Immunologic control of a parasitic arthropod. Identification of a protective antigen from Boophilus microplus. J Immunol 143:1346-1351

Willadsen P, Bird P, Cobon GS et al (1995) Commercialisation of a recombinant vaccine against Boophilus microplus. Parasitology 110(Suppl):S43-S50

Willadsen P, Smith D, Cobon G et al (1996) Comparative vaccination of cattle against Boophilus microplus with recombinant antigen Bm86 alone or in combination with recombinant Bm91. Parasite Immunol 18:241-246

Wu Y, Ellis RD, Shaffer D et al (2008) Phase 1 trial of malaria transmission blocking vaccine candidates Pfs 25 and Pvs 25 formulated with montanide ISA 51. PLoS One 3:e2636

Yun SI, Lee YM (2014) Japanese encephalitis: the virus and vaccines. Hum Vaccin Immunother 10:263-279

Zhang P, Tian Z, Liu G et al (2011) Characterization of acid phosphatase from the tick Haemaphysalis longicornis. Vet Parasitol 182:287-296

Zhou J, Liao M, Hatta T et al (2006a) Identification of a follistatinrelated protein from the tick Haemaphysalis longicornis and its effect on tick oviposition. Gene 372:191-198

Zhou J, Ueda M, Umemiya R et al (2006b) A secreted cystatin from the tick Haemaphysalis longicornis and its distinct expression patterns in relation to innate immunity. Insect Biochem Mol Biol 36:527-535 